\title{
Trends in Incidence of Urinary Tract Infection in Mainland China from 1990 to 2019
}

\author{
Sheng Yuan ${ }^{1,2}$ \\ Ying Shi ${ }^{3}$ \\ Minmin $\mathrm{Li}^{4}$ \\ Xiaojun $\mathrm{Hu}^{5}$ \\ Ruhai Bai $\mathbb{D D}^{6}$ \\ 'Department of New Medicine and \\ Needle Pricking, The First Affiliated \\ Hospital of Jinan University, Guangzhou, \\ Guangdong, People's Republic of China; \\ ${ }^{2}$ Department of Surgery, The First \\ Affiliated Hospital of Guangzhou \\ University of Chinese Medicine, \\ Guangzhou, Guangdong, China; ${ }^{3}$ School \\ of Public Health, Shaanxi University of \\ Chinese Medicine, Xi'an, Shaanxi, \\ People's Republic of China; ${ }^{4}$ Department \\ of Infection Disease Control and \\ Prevention, Shaanxi Provincial Center for \\ Disease Control and Prevention, Xi'an, \\ Shaanxi, People's Republic of China; \\ ${ }^{5}$ Department of Hepatobiliary Surgery, \\ The Fifth Affiliated Hospital of Southern \\ Medical University, Guangzhou, \\ Guangdong, People's Republic of China; \\ ${ }^{6}$ School of Public Affair, Nanjing \\ University of Science and Technology, \\ Nanjing, Jiangsu, People's Republic of \\ China
}

Purpose: Urinary tract infection (UTI) is the second-most-common type of infection in China. This study aimed to determine the long-term trends in the incidence of UTI in Mainland China between 1990 and 2019.

Materials and Methods: Data were extracted from the Global Burden of Disease Study 2019 and were analyzed with the age-period-cohort framework.

Results: The net drift in the incidence of UTI was $-0.37 \%$ (95\% CI: $-0.40 \%,-0.35 \%)$ in males and $-0.25 \%(95 \% \mathrm{CI}:-0.29 \%,-0.20 \%)$ in females. For males, the local drift was lower than $0(\mathrm{P}<0.05)$ among those younger than 90 years. For females, the local drift was lower than $0(\mathrm{P}<0.05)$ among those younger than 60 years and higher than $0(\mathrm{P}<0.05)$ in those aged 65-79 years. In the same birth cohort, the incidence of UTI was higher in females than in males in all age groups $(\mathrm{P}<0.05)$. The period relative risk $(\mathrm{RR})$ showed a decreasing pattern after 2005 in both sexes. The cohort RR showed a downward trend of the birth cohort after 1905 for males and 1960 for females.

Conclusion: The incidence has increased significantly among older females over the past 30 years. It is necessary to develop a comprehensive intervention plan for reproductive health services covering females and males of all ages.

Keywords: age-period-cohort analysis, urinary tract infection, bacterial infection

\section{Introduction}

A urinary tract infection (UTI) normally refers to a bacterial infection that occurs between the urethral orifice and the perirenal fascia. ${ }^{1}$ It is one of the most common types of bacterial infection, ${ }^{2}$ with 150 million people worldwide affected annually. ${ }^{3}$

Urinary tract infections (UTIs) have a serious economic burden on society by harming work efficiency, personal and family responsibilities, quality of life, and sexual health. ${ }^{4-7}$ At the same time, UTIs have high recurrence rates. ${ }^{2,8}$ The repeated occurrence of UTIs can cause serious sequelae, including urinary sepsis, glandular cystitis, and pyelonephritis, ${ }^{9,10}$ and even severe renal failure leading to death. ${ }^{11}$ The frequent or improper use of antibiotics when treating UTIs can also cause complications, such as high antibiotic resistance, dysbacterial flora, and Clostridium difficile colitis. ${ }^{12}$ For females, UTIs during pregnancy can cause serious complications of pregnancy, parturient and perinatal infants, such as premature birth, eclampsia, anemia, and low-birthweight infants. ${ }^{13}$

China is currently the most populous country globally, and UTI imposes a huge burden on the health system. As the second-most-common type of infection in China, UTI accounted for $11.65 \%$ of healthcare-associated infections from 2006 to $2016 .^{14}$ However, there have been few studies of the trends in the incidence of UTI in China.
School of Public Affair, Nanjing University of Science and Technology, No. 200, Xiao Lingwei Street, Nanjing, Jiangsu, People's Republic of China

Email ruhaibai@hotmail.com 
This study used data from the Global Burden of Disease Study 2019 (GBD 2019) data and applied an age-periodcohort (APC) framework to explore the incidence of UTI according to sex and age groups in Mainland China over the past 30 years. The findings of this research will fill major gaps in this area and may also be useful as reference information when planning future inventions for UTI in Mainland China.

\section{Materials and Methods}

\section{Data Source}

The GBD 2019 database contains internally consistent estimates of the sex- and age-specific incidence rates of more than 300 diseases and injury types globally, regionally, and nationally from 1990 to $2019 .{ }^{15}$ GBD 2019 data on Mainland China mainly come from five sources: the Chinese Center for Disease Control and Prevention, the National Office for Maternal and Child Health Surveillance, the Center for Health Statistics and Information at the National Health Commission, the National Office for Cancer Control and Prevention, and individual researchers at academic institutions worldwide. ${ }^{16}$ UTI was defined in this study according to the 9th and 10th revisions of the International Classification of Diseases (ICD10: N10-N12.9, N13.6, N15, N15.1-N16.8, N30-N30.3, N30.8-N30.9, N34-N34. 3, N39.0-N39.2; ICD9: 590-590.9, 595-595.9, 597-597.9, 599.0). ${ }^{15}$ The datasets generated and/or analyzed during the current study are available in the GBD repository: http://ghdx.healthdata.org/gbd-results-tool.

\section{Statistical Analysis}

An APC model was used in this study to evaluate the effects of age, period, and cohort on the incidence of UTI. The APC is widely used in epidemiology to identify secular trends in disease incidence and mortality rate. The APC can be considered essentially as a multiple regression model which was developed based on the Poisson distribution. The specific equation was exhibited as follows:

$$
Y=\mu+\alpha \text { Age }_{i}+\text { Beriod }_{j}+\gamma \text { Cohort }_{k}+\varepsilon
$$

where $Y$ denotes the response variable- the net effect on UTI incidence for groups, $\alpha, \beta$, and $\gamma$ denoted the coefficient of age, period, and cohort of the APC model, respectively, and $\mu$ denoted the intercept of the model, and $\varepsilon$ denoted the residual of the APC model.

The age effect represents how the risks of different outcomes vary between age groups. The period effect represents the change in the results over time and affects all age groups at the same time. The cohort effect is related to the differences in results between groups with the same birth year. This study focused on estimating the following parameters: the net drift, which indicates the overall annual percentage change; the local drift, which indicates annual percentage changes for each age group; the longitudinal age curve, which indicates the fitted longitudinal age-specific rates in the reference cohort adjusted for period deviations; and the period (or cohort) relative risk (RR), which is the RR of a period (or cohort) relative to the reference period (or cohort) adjusted for age and nonlinear cohort (or period) influences.

Figure 1 shows the flow chart presenting the procedures of this study. To conduct the APC analysis, the

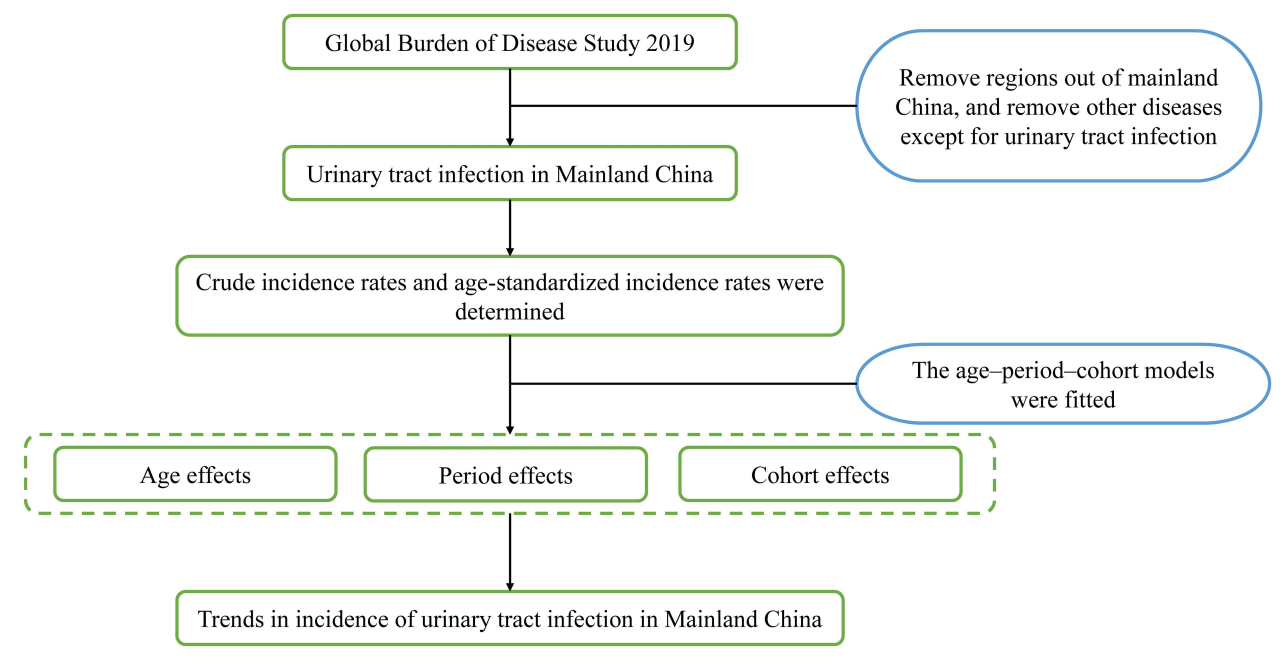

Figure I Study flowchart. 


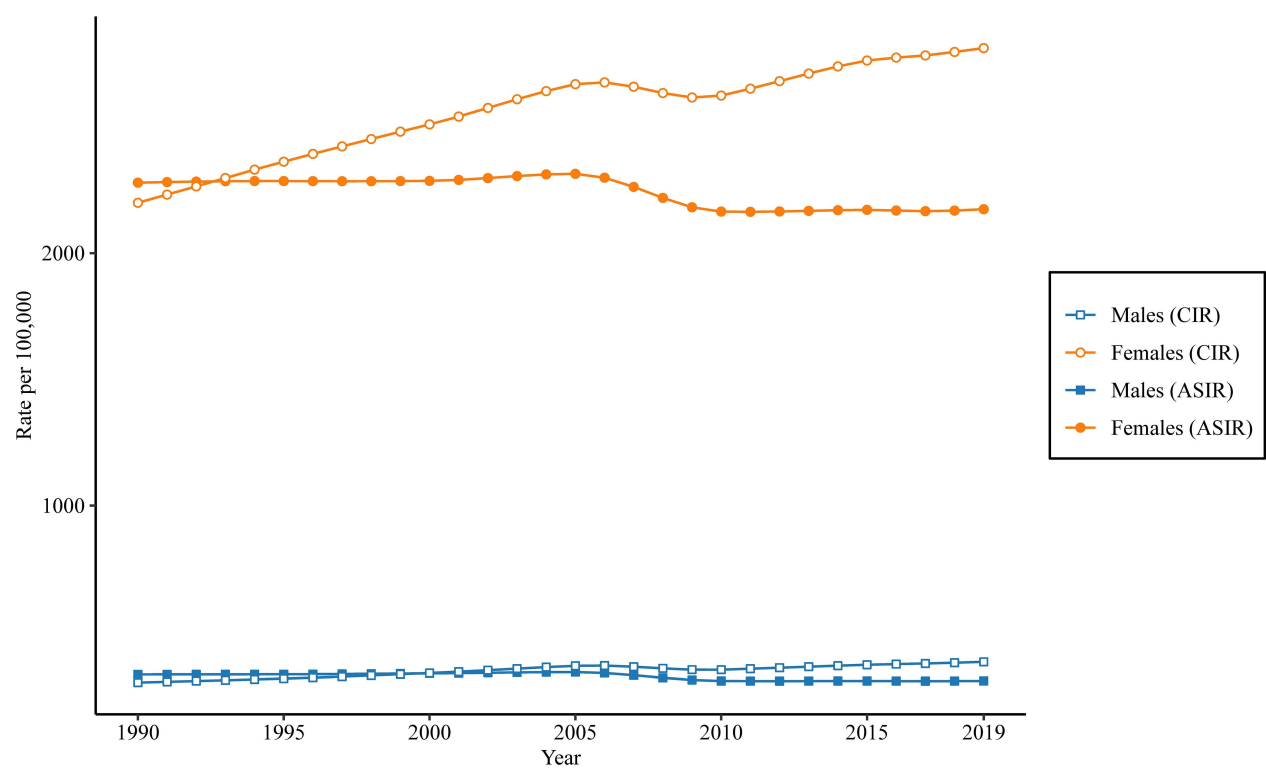

Figure 2 Trends in the age-standardized incidence rates (ASIRs) and crude incidence rates (CIRs) per 100,000 population for UTI by sex in Mainland China from I990 to 2019. The GBD 2019 global age-standard population was used to assess the age-standardized incidence rate.

incidence data were grouped into consecutive 5-year periods from 1990 to 2019. Successive 5-year age intervals from 0 to 4 years to 90 to 94 years were included. Individuals aged $>95$ years were not considered in this study because those aged $>95$ years are recorded as a single group in the GBD 2019 database. The parameter was estimated using the APC Web Tool ${ }^{17}$ (Biostatistics Branch, National Cancer Institute, Bethesda). The center value of the age group, age, and birth cohort was defined as the corresponding reference. ${ }^{18}$ Wald chi-square tests were used to test the significance of the functions that could be estimated. The GBD 2019 global age-standard population was used to assess the age-standardized incidence rate (ASIR). All statistical tests were two-sided, and differences were considered statistically significant for $P$ values less than 0.05 .

\section{Results}

\section{Trends in the Incidence Rates of UTI by Sex from 1990 to 2019}

The crude incidence rate (CIR) and ASIR of UTI from 1990 to 2019 according to sex are shown in Figure 2. For males, the CIR of UTI increased from 298.6 per 100,000 in 1990 to 380.8 per 100,000 in 2019. The standardized incidence of male UTI decreased, from 331.0 per 100,000 in 1990 to 305.0 per 100,000 in
2019. For females, the CIR of UTI increased from 2199.4 per 100,000 in 1990 to 2812.3 per 100,000 in 2019. The standardized incidence of UTI among females also declined, from 2279.1 per 100,000 in 1990 to 2173.9 per 100,000 in 2019. Overall, from 1990 to 2019 the CIR of UTI in Mainland China increased by $27.5 \%$ in males and by $27.9 \%$ in females, while the standardized rate of UTI decreased by $7.8 \%$ in males and by $4.6 \%$ in females.

\section{Local Drift with Net Drift Values for the Incidence of UTI}

The overall annual change percentage (net drift) of the incidence of UTI and the annual change in UTI in each age group (local drift) are shown in Figure 3. The net drift was $-0.37 \%(95 \%$ confidence interval $[\mathrm{CI}]=-$ $0.40 \%$ to $-0.35 \%)$ for males and $-0.25 \%(95 \% \mathrm{CI}=-$ $0.29 \%$ to- $0.20 \%$ ) for females, all showed a significant decrease over the past 30 years $(\mathrm{P}<0.05)$. The local drift among females younger than 60 years was significantly decreased $(\mathrm{P}<0.05)$ and was lowest among those aged $0-5$ years, at $-1.25 \%(95 \%$ CI $=-1.53 \%$ to- $0.98 \%)$. The local drift of females aged 65-79 years was significantly increased $(\mathrm{P}<0.05)$. The local drift of males aged younger than 90 years decreased significantly $(\mathrm{P}<0.05)$, and was lowest among those aged $0-5$ years, at $-1.13 \%(95 \% \mathrm{CI}=-1.20 \%$ to $-1.05 \%)$. 


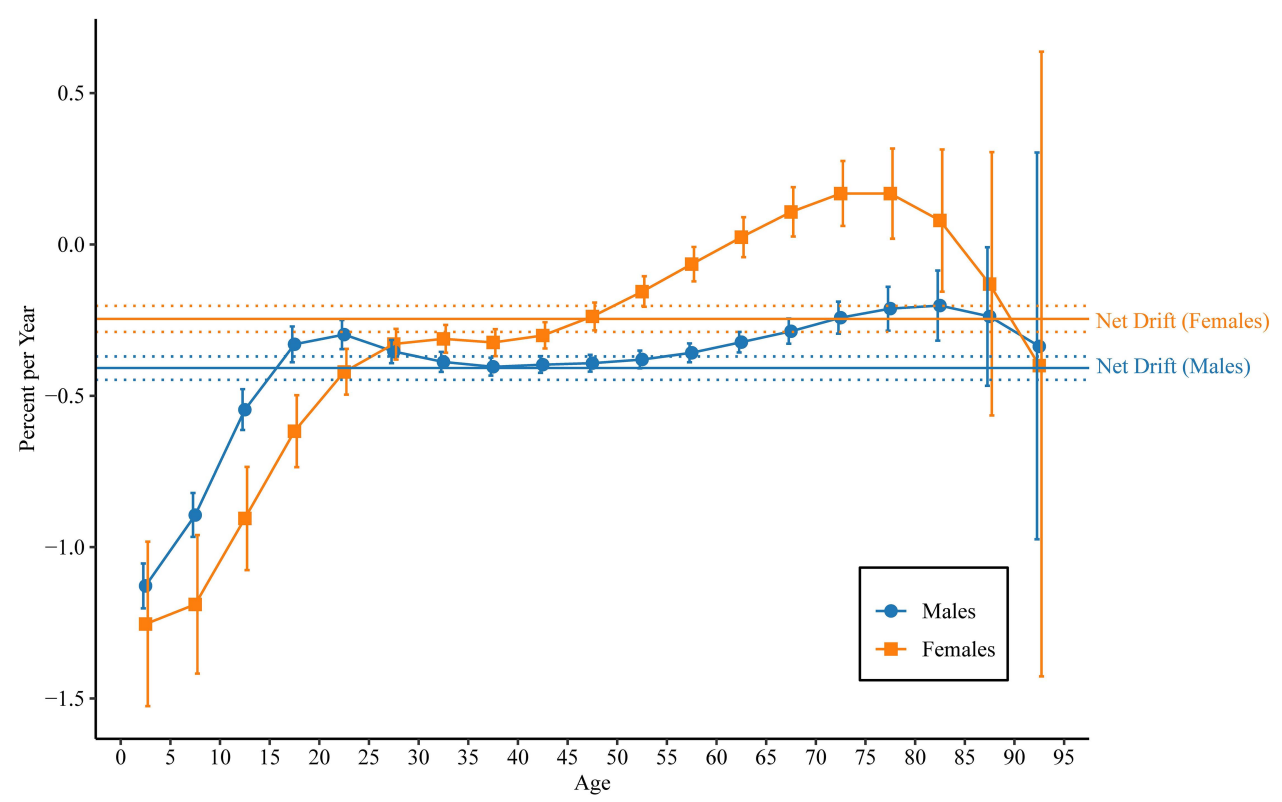

Figure 3 Local drift with net drift values for the incidence of UTI in Mainland China. Age-group-specific annual percentage change (local drift) with the overall annual percentage change (net drift) in the incidence rates of UTI and the corresponding $95 \%$ confidence intervals $(\mathrm{Cls})$.

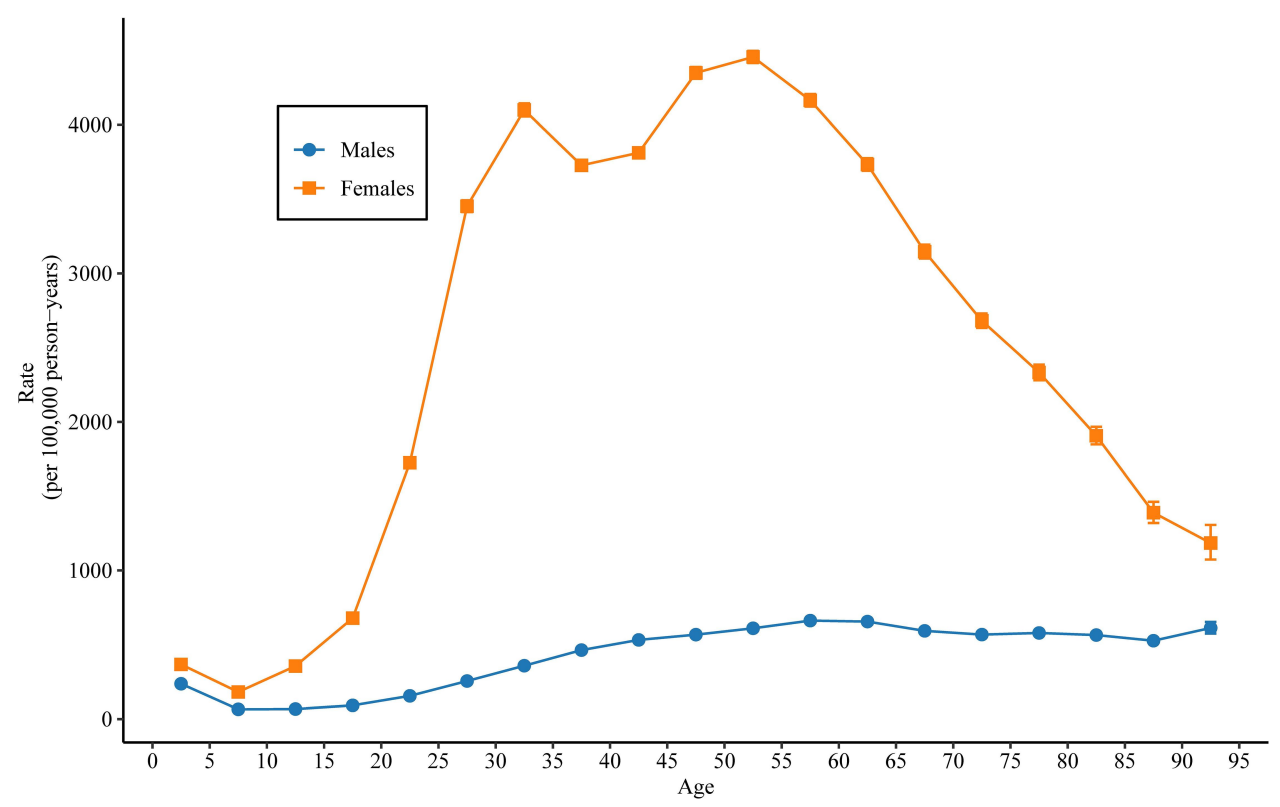

Figure 4 Longitudinal age curves of the incidence of UTI in Mainland China. Fitted longitudinal age-specific rates of the incidence of UTI (per I00,000 person-years) and the corresponding $95 \% \mathrm{Cls}$ (some of them were too narrow to show in the figure).

\section{Longitudinal Age Curves of Incidence of UTI by Sex}

Figure 4 shows longitudinal age curves of the incidence of UTI. In the same birth cohort, females aged 5-9 years had the lowest incidence of UTI. For females, the incidence of UTI increased rapidly with age, reaching the first peak at
30-34 years, with a second peak at 50-54 years. For males, with the exception of children aged $0-4$ years, UTI first increased and then decreased with age, with their incidence being highest between 55 and 59 years of age. Also, the incidence of UTI was significantly higher in females than in males for all age groups $(\mathrm{P}<0.05)$. 

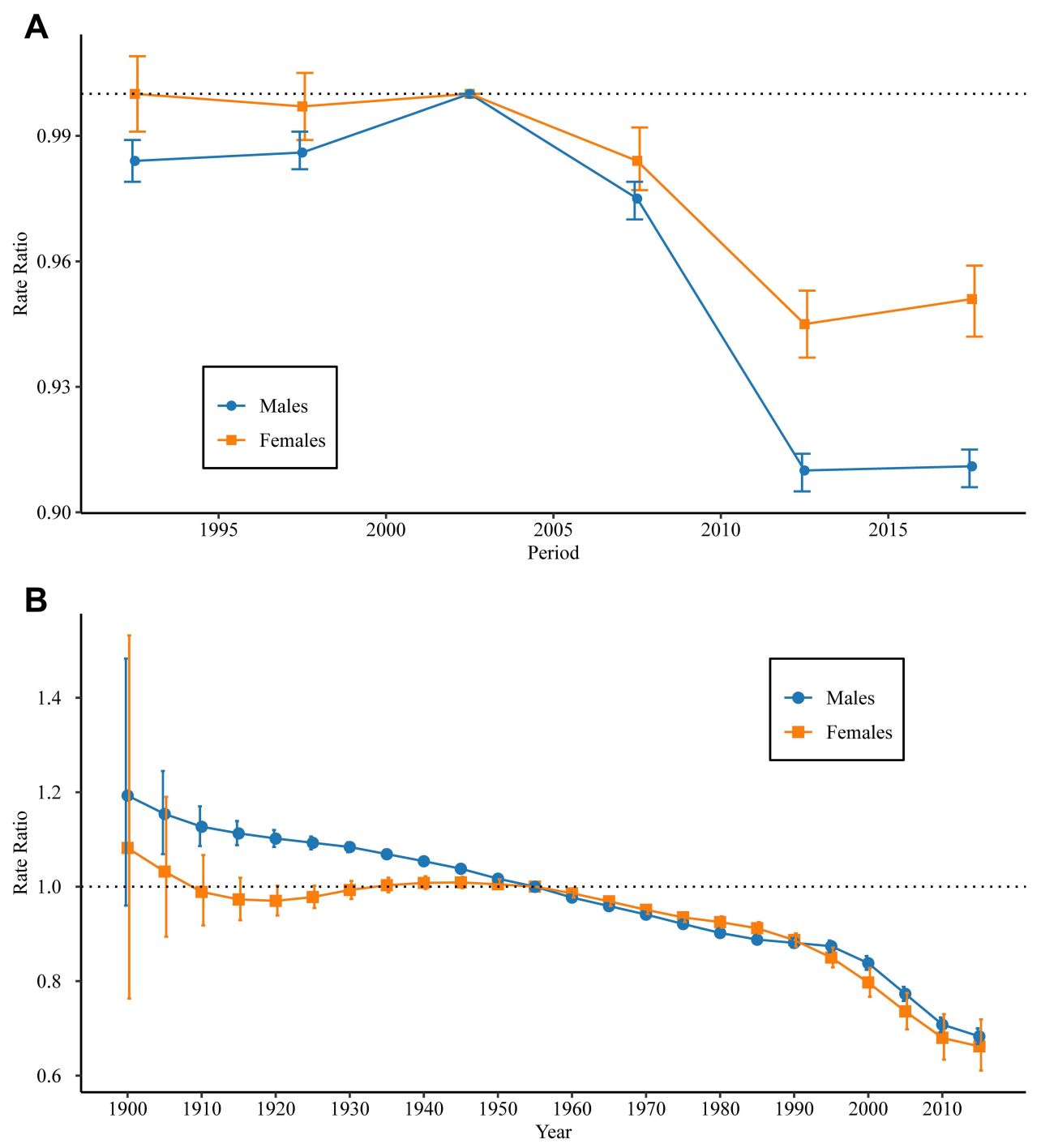

Figure 5 Period and cohort relative risks (RRs) of the incidence rate of UTI by sex in Mainland China. (A) For period RR and (B) for cohort RR. The period RR of each period compared with the reference (2000 to 2004) adjusted for age and nonlinear cohort effects, and the corresponding 95\% Cls. The cohort RR of each cohort compared with the reference (the 1955 cohort) adjusted for age and nonlinear period effects, and the corresponding $95 \% \mathrm{Cls}$ (some of them were too narrow to show in the figure).

\section{Period and Cohort Relative Risks of Incidence Rate of UTI by Sex}

Figure 5 shows the estimated period RRs and cohort RRs according to sex. Compared with the reference period from 2000 to 2004, period RRs have declined in recent years in both females and males. The cohort RRs showed a general decline after 1960 in females and after 1905 in males.

\section{Discussion}

This study used the APC framework to explore the longterm trends in the incidence of UTI in Mainland China from 1990 to 2019. As far as we know, this is the first study to investigate APC-specific effects on the incidence of UTI according to sex. Our results show that between
1990 and 2019, the ASIR of UTI decreased in both Chinese males and females, whereas the incidence of UTI increased in females aged 65 to 79 years.

Our incidence estimates on older females are broadly consistent with previous population-based studies in the UK. In the Clinical Practice Research Datalink study (Approximately 7\% of the UK population are included), the incidence of UTI for female increased (episodes per 100 person-years at risk) from 9.03 to 10.96 in those aged $65-74$, and 11.35 to 14.34 in those aged 75-84 from 2004 to $2014 .^{19}$ In contrast to previous studies, we did not identify the increase in any age groups in male for the last 30 years. Reasons for this could include the increased focus in China on reproductive health in recent years and lifestyle changes due to economic development. 
This study found certain differences between the CIR and ASIR of UTI, which may be related to recent changes in the population structure of China. During the past 30 years, the decline in the fertility of the Chinese population and the increased life expectancy have together produced a continual decline in the proportion of children younger than 14 years. ${ }^{20}$ According to a national survey conducted by the National Bureau of Statistics, as at the end of 2018, the number of older adults aged at least 60 years in China had reached 249 million, accounting for approximately $17.9 \%$ of the total population. ${ }^{21}$

This study found that the incidence of UTI was higher in females than in males. America found the same situation that the lifetime risk of UTI for females is about $50 \%$ and about $20 \%$ for males. ${ }^{22,23}$ In the absence of known urinary tract abnormalities, females are more likely to develop UTIs than males due to the shortness of the female urethra, interference of urine flow, and frequent vaginal colonization. ${ }^{24}$ The present study found that the incidence of UTI decreased between 1990 and 2019 in females younger than 60 years and in males younger than 90 years. For females, this may be related to improvements in reproductive health in China. ${ }^{25}$ In 2003, China launched a maternal health system covering the rural population that included providing females in central and western regions with economic subsidies for delivery in hospitals, as well as providing all citizens with free selected reproductive health services, which may have reduced the probability of UTIs in young females. However, the reproductive health services in China focus on young people, which means that older females - and especially those living in rural areas - often cannot receive free services. ${ }^{26}$ Considering the increasing incidence of UTI among Chinese older females over the past 30 years, it is necessary to develop a comprehensive intervention plan for reproductive health services covering older females. ${ }^{26}$

For males, the decrease in UTIs among those younger than 90 years over the past 30 years may be related to lifestyle changes brought about by economic development. Moreover, the increased focus in China on male reproductive health in recent years has also helped to reduce UTIs in younger groups. Like most countries worldwide, the Chinese population is rapidly aging. ${ }^{21}$ It is estimated that by 2030 the elderly population in China will reach 400 million, equivalent to the total population of 15 European Union countries, ${ }^{27}$ and China will remain the most populous country in the world and the country with the largest elderly population. ${ }^{27}$ Considering the increasing trend in UTIs among Chinese older females over the past
30 years, such infections are likely to have a huge impact on the health of Chinese elderly in the future.

Age is an important risk factor for UTI. This study found that the incidence of UTI was higher in children aged $0-4$ years than in older children. UTI is the most common bacterial infection in childhood ${ }^{28}$ and the main reason for hospitalization. ${ }^{29}$ The higher incidence of UTI in childhood may be related to differences in the bacterial intestinal flora of children, an immature immune system, and an abnormal urinary tract. ${ }^{29}$ It should be noted that the occurrence of UTI in children may be a sentinel event of potential kidney damage, and hence needs to receive sufficient clinical attention.

The higher incidence of UTI in young people may be related to more-active sexual behavior. ${ }^{30}$ For elderly females, a significant decrease in estrogen levels after menopause will cause some physiological changes in normal females, such as vaginal atrophy, changes in the normal vaginal flora, and an increase in the vaginal $\mathrm{pH}$. These physiological changes are related to the occurrence of recurrent UTIs. ${ }^{31}$ At the same time, perimenopausal females are more susceptible to bacterial infections and UTIs. ${ }^{32}$

The period effect reflects the immediate impact of social factors on the incidence of disease. ${ }^{33}$ The present study found that the risk of UTI in both males and females decreased after 2005 compared with 2000 to 2004 . The reduced risk of male UTI may be related to greater attention being paid to male reproductive health in China in recent years. Since 2000, China has designated 28 October each year as Men's Health Day. By identifying different themes and holding special exhibitions on male health each year, the Chinese government has promoted their health issues and announced a series of public policies conducive to promoting male reproductive health.

As mentioned above, the risk of female UTI has reduced, which is mainly related to improvements in reproductive health. The cohort effect reflects changes in disease risk in a particular birth cohort. ${ }^{33}$ In the present study, the birth cohort effect showed that both males and females born more recently had lower risks of UTI. This may be related to changes in lifestyles, more-sanitary conditions, and improvements in health habits.

This study has revealed the trends in the incidence of UTI in Mainland China over the past 30 years. The results obtained in this study may help in controlling the problem of UTI in China. However, this study also had some limitations. First, the study utilized GBD data on UTI in Mainland China. These data are macrolevel estimates at the national level, and it might not be possible to reliably interpret macrolevel results at the individual level. Secondly, this study did not compare the trends in 
the incidence rate of UTI between urban and rural areas of China due to data limitations. Thus, the epidemiology of UTI in urban and rural areas needs to be further analyzed in the future.

\section{Conclusion}

In conclusion, the overall incidence of UTI among Chinese males and females has decreased over the past 30 years, whereas the incidence has increased significantly among older females. Considering the continued aging of the Chinese population, UTI is bound to become an important health problem affecting the health of the elderly in Mainland China. It is necessary to develop a comprehensive intervention plan for reproductive health services covering females and males of all ages.

\section{Ethics Statement}

The GBD 2019 data are freely available to the world's researchers and policymakers (http://ghdx.healthdata.org/ gbd-results-tool). The GBD 2019 uses de-identified, aggregated data, therefore a waiver of informed consent was reviewed and approved by the University of Washington Institutional Review Board.

\section{Acknowledgments}

This work was

funded by project of Administration of TraditionalChinese Medicine ofGuangdong Province of China (NO. 20191091). We are grateful to the individuals who participated in the study.

\section{Disclosure}

The authors report no conflicts of interest in this work.

\section{References}

1. Barnett BJ, Stephens DS. Urinary tract infection: an overview. $\mathrm{Am}$ J Med Sci. 1997;314(4):245-249.

2. Foxman B. The epidemiology of urinary tract infection. Nat Rev Urol. 2010;7(12):653-660. doi:10.1038/nrurol.2010.190

3. McLellan LK, Hunstad DA. Urinary tract infection: pathogenesis and outlook. Trends Mol Med. 2016;22(11):946-957. doi:10.1016/j. molmed.2016.09.003

4. Renard J, Ballarini S, Mascarenhas T, et al. Recurrent lower urinary tract infections have a detrimental effect on patient quality of life: a prospective, observational study. Infect Dis Ther. 2014;4 (1):125-135. doi:10.1007/s40121-014-0054-6

5. Ciani O, Grassi D, Tarricone R. An economic perspective on urinary tract infection: the "costs of resignation". Clin Drug Investig. 2013;33 (4):255-261. doi:10.1007/s40261-013-0069-x
6. Keating KN, Perfetto EM, Subedi P. Economic burden of uncomplicated urinary tract infections: direct, indirect and intangible costs. Expert Rev Pharmacoecon Outcomes Res. 2005;5(4):457-466. doi:10.1586/14737167.5.4.457

7. Schaeffer AJ, Nicolle LE. Urinary tract infections in older men. $N$ Engl J Med. 2016;374(22):2192. doi:10.1056/NEJMcp 1503950

8. Nagamatsu K, Hannan T, Guest R, et al. Dysregulation of Escherichia coli $\alpha$-hemolysin expression alters the course of acute and persistent urinary tract infection. Proc Nat Acad Sci USA. 2015;112(8):E871E880. doi:10.1073/pnas.1500374112

9. Zhiqiang C, Zhangqun Y, Wei Z. Clinical investigation on the correlation between lower urinary tract infection and cystitis glandularis. J Huazhong Univ Sci Technol. 2004;24(3):303-304. doi:10.1007/ BF02832021

10. Andreini P, Bonechi S, Bianchini M, Garzelli A, Mecocci A. Automatic image classification for the urinoculture screening. Comput Biol Med. 2016;70:12-22. doi:10.1016/j.compbiomed.2015.12.025

11. Abbott K, Swanson S, Richter E, et al. Late urinary tract infection after renal transplantation in the United States. Am J Kidney Dis. 2004;44(2):353-362. doi:10.1053/j.ajkd.2004.04.040

12. Flores-Mireles AL, Walker JN, Caparon M, Hultgren SJ. Urinary tract infections: epidemiology, mechanisms of infection and treatment options. Nat Rev Microbiol. 2015;13(5):269-284. doi:10.1038/ nrmicro3432

13. Le J, Briggs G, McKeown A, Bustillo G. Urinary tract infections during pregnancy. Ann Pharmacother. 2004;38(10):1692-1701. doi:10.1345/aph.1D630

14. Wang J, Liu F, Tartari E, et al. The prevalence of healthcare-associated infections in Mainland China: a systematic review and meta-analysis. Infect Control Hosp Epidemiol. 2018;39 (6):701-709. doi:10.1017/ice.2018.60

15. GBD 2019 Diseases and Injuries Collaborators. Global burden of 369 diseases and injuries in 204 countries and territories, 1990-2019: a systematic analysis for the Global Burden of Disease Study 2019. Lancet. 2020;396(10258):1204-1222.

16. Zhou M, Wang H, Zeng X, et al. Mortality, morbidity, and risk factors in China and its provinces, 1990-2017: a systematic analysis for the Global Burden of Disease Study 2017. Lancet. 2019;394 (10204):1145-1158. doi:10.1016/S0140-6736(19)30427-1

17. Rosenberg PS, Check DP, Anderson WF. A web tool for age-periodcohort analysis of cancer incidence and mortality rates. Cancer Epidemiol Biomarkers Prev. 2014;23(11):2296-2302. doi:10.1158/ 1055-9965.EPI-14-0300

18. Wang $\mathrm{P}, \mathrm{Xu} \mathrm{C}, \mathrm{Yu} \mathrm{C}$. Age-period-cohort analysis on the cancer mortality in rural China: 1990-2010. Int $J$ Equity Health. 2014;13:1. doi:10.1186/1475-9276-13-1

19. Ahmed H, Farewell D, Jones HM, Francis NA, Paranjothy S, Butler CC. Incidence and antibiotic prescribing for clinically diagnosed urinary tract infection in older adults in UK primary care, 2004-2014. PLoS One. 2018;13(1):e0190521. doi:10.1371/journal.pone.0190521

20. Wei Y, Wang Z, Wang H, Li Y, Jiang Z. Predicting population age structures of China, India, and Vietnam by 2030 based on compositional data. PLoS One. 2019;14(4):e0212772-e0212772. doi:10.1371/ journal.pone. 0212772

21. He X, Song M, Qu J, et al. Basic and translational aging research in China: present and future. Protein Cell. 2019;10(7):476-484. doi:10.1007/s13238-019-0617-0

22. Griebling TL. Urologic diseases in America project: trends in resource use for urinary tract infections in women. $J$ Urol. 2005;173(4):1281-1287. doi:10.1097/01.ju.0000155596.98780.82

23. Griebling TL. Urologic diseases in america project: trends in resource use for urinary tract infections in men. $J$ Urol. 2005;173 (4):1288-1294. doi:10.1097/01.ju.0000155595.98120.8e 
24. Gupta K, Grigoryan L, Trautner B. Urinary Tract Infection. Ann Intern Med. 2017;167(7):ITC49-ITC64. doi:10.7326/AITC201710030

25. Fang J. Commentary: China's changing health system: implications for sexual and reproductive health. Glob Public Health. 2015;10 (2):249-251. doi:10.1080/17441692.2014.986171

26. Sun X, Shu X, Zong Z, Mao J, Sun Y, Hearst N. Unmet sexual and reproductive health needs of women aged 50 to 64 years in rural China. Menopause. 2015;22(5):505-511. doi:10.1097/GME.0000000000000346

27. Sun R, Cao H, Zhu X, Liu J-P, Dong E. Current aging research in China. Protein Cell. 2015;6(5):314-321. doi:10.1007/s13238-015-0145-5

28. Simoes e Silva AC, Oliveira EA. Update on the approach of urinary tract infection in childhood. J Pediatr (Rio J). 2015;91(Suppl 6):S2S10. doi:10.1016/j.jped.2015.05.003

29. Kutasy B, Coyle D, Fossum M. Urinary tract infection in children: management in the era of antibiotic resistance-a pediatric urologist's view. Eur Urol Focus. 2017;3(2-3):207-211. doi:10.1016/j. euf.2017.09.013
30. Tan CW, Chlebicki MP. Urinary tract infections in adults. Singapore Med J. 2016;57(9):485-490. doi:10.11622/smedj.2016153

31. Raz R, Stamm WE. A controlled trial of intravaginal estriol in postmenopausal women with recurrent urinary tract infections. $N$ Engl J Med. 1993;329(11):753-756. doi:10.1056/NEJM199309093291102

32. Guglietta A. Recurrent urinary tract infections in women: risk factors, etiology, pathogenesis and prophylaxis. Future Microbiol. 2017;12:239-246. doi:10.2217/fmb-2016-0145

33. Yang J, Zhang Y, Luo L, Meng R, Yu C. Global mortality burden of cirrhosis and liver cancer attributable to injection drug use, 1990-2016: an age-period-cohort and spatial autocorrelation analysis. Int J Environ Res Public Health. 2018;15(1).

\section{Publish your work in this journal}

The International Journal of General Medicine is an international, peer-reviewed open-access journal that focuses on general and internal medicine, pathogenesis, epidemiology, diagnosis, monitoring and treatment protocols. The journal is characterized by the rapid reporting of reviews, original research and clinical studies across all disease areas. The manuscript management system is completely online and includes a very quick and fair peer-review system, which is all easy to use. Visit http://www.dovepress.com/ testimonials.php to read real quotes from published authors. 